\title{
Wireless Non-contact Cardiac and Neural Monitoring
}

\author{
Yu M. Chi, Patrick Ng \\ Department of Electrical and \\ Computer Engineering \\ University of California, San \\ Diego \\ 9500 Gilman Drive \\ La Jolla, CA 92093 \\ m1chi, png@ucsd.edu
}

\author{
Eric Kang, Joseph Kang \\ Department of Bioengineering \\ University of California, San \\ Diego \\ 9500 Gilman Drive \\ La Jolla, CA 92093 \\ ekang4@ucsd.edu
}

Gert Cauwenberghs
Department of Bioengineering
University of California, San
Diego
9500 Gilman Drive
La Jolla, CA 92093
gert@ucsd.edu

\author{
Jennifer Fang \\ Department of Computer \\ Science and Engineering \\ University of California, San \\ Diego \\ 9500 Gilman Drive \\ La Jolla, CA 92093 \\ jyfang@ucsd.edu
}

\begin{abstract}
Ubiquitous physiological monitoring will be a key driving force in the upcoming wireless health revolution. Cardiac and brain signals in the form of ECG and EEG are two critical health indicators that directly benefit from long-term monitoring. Despite advancements in wireless technology and electronics miniaturization, however, the use of wireless home ECG/EEG monitoring is still limited by the inconvenience and discomfort of wet adhesive electrodes.

We have developed a wireless biopotential instrumentation system using non-contact capacitive electrodes that operate without skin contact. The sensors can be embedded within comfortable layers of fabric for unobtrusive use. All of the issues relating to the design of low noise, high performance capacitive sensors are discussed along with full technical details, circuit schematics and construction techniques.

The non-contact electrode has been integrated into both a wearable ECG chest harness as well a EEG headband. We have also designed a compact, battery-powered, wireless data acquisition system to interface with multiple electrodes and monitor patient cardiac and neural signals in real time. Experimental data shows that the non-contact capacitive electrode perform comparable to $\mathrm{Ag} / \mathrm{AgCl}$ electrodes using our special chest harness and head bands to ensure tight, movement-free electrode positioning.
\end{abstract}

\section{General Terms}

EEG, ECG, Wireless Health, Capacitive Sensors

Permission to make digital or hard copies of all or part of this work for personal or classroom use is granted without fee provided that copies are not made or distributed for profit or commercial advantage and that copies bear this notice and the full citation on the first page. To copy otherwise, to republish, to post on servers or to redistribute to lists, requires prior specific permission and/or a fee.

Wireless Health 2010, October 5-7, 2010, San Diego, USA

Copyright 2010 ACM 978-1-60558-989-3 ...\$10.00.

\section{INTRODUCTION}

Brain and cardiac biopotential signals in the form of EEG and ECG are two critical physiological indicators that are directly suited for long-term wireless health monitoring. Yet despite advancements in wireless technology and electronics miniaturization, however, the use EEG/ECG has still been largely limited by the inconvenience and discomfort of conventional wet contact electrodes.

For home use, clinical grade adhesive electrodes are often cited as irritating and uncomfortable leading to low usage compliance. As an alternative, dry electrodes [1] [2] have started becoming much more common-place. However, like wet electrodes, dry electrodes still require direct electrical contact to the skin. In addition, dry electrodes, which do not have the benefit of a conductive gel, are much more sensitive to the condition of the skin and are highly susceptible to motion artifacts. For future wireless health systems, a less obtrusive sensor is needed to match the advancements made in wireless technology.

In contrast to wet and dry contact sensors, non-contact capacitive electrodes do not require an ohmic connection to the body. This offers numerous advantages since noncontact electrodes require zero preparation, are completely insensitive to skin conditions and can be embedded inside a garment for a completely unobtrusive, patient-friendly system. While the concept of non-contact biopotential sensors is not new, with the first working device reported decades ago [3], a practical device for patient use has yet to materialize. More recently, several authors have presented results from designs utilizing the latest in commercially available discrete low noise amplifiers [4] [5] [6], including some wireless designs [7].

Over the years, many clever designs have appeared, some proprietary. However, nothing has really progressed beyond the 'lab prototype' stage. In addition, general knowledge about capacitive sensors and how to design and construct them are scarce in literature. In this paper, we attempt to address these shortcomings by presenting the full designs, including all the relevant details in the analog front-end, 


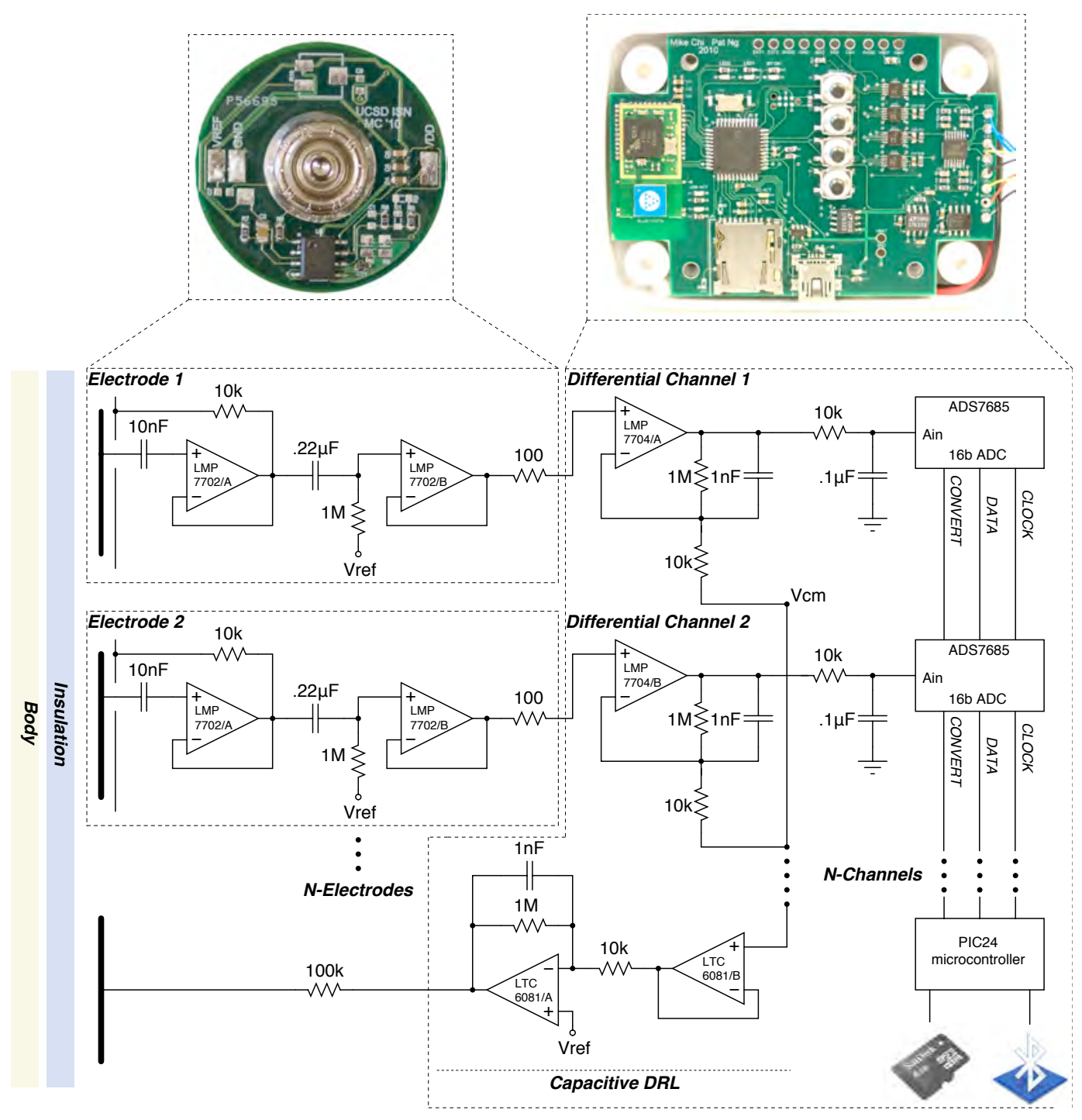

Figure 1: Full schematic of wireless ECG/EEG system. The capacitive electrode PCB contains the front-end amplifier. Differential gain, digitization, active grounding and digital processing/wireless is contained on separate base unit.
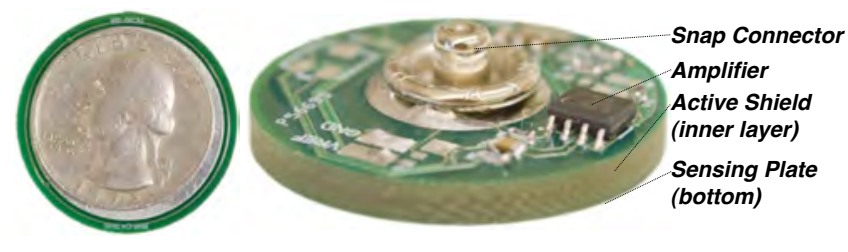

Figure 2: Picture of the non-contact, capacitive electrode. The sensor is manufactured on a standard PCB, which contains the amplifier circuits on the top and the sensing plate on the bottom.

for a high-quality, contactless, wireless ECG/EEG monitor. In addition, we characterize the system-level performance by directly comparing the capacitive electrode against traditional, clinical $\mathrm{Ag} / \mathrm{AgCl}$ electrodes and show that they perform equally well in many applications.

\section{SYSTEM DESIGN}

A full schematic depicting the wireless, non-contact sensor system is shown in Figure 1. Each capacitive electrode contains an onboard amplifier, filter, buffer and connects to the wireless base unit. The full operation of the capacitive electrode's amplifier and analog front-end, including circuit/noise theory, has been well described in previous publications [8] [9]. In this paper, we focus on the system-level design and results from our latest generation, optimized sensor.

All of the electrodes, including the active ground, can be fully insulated. Since no galvanic connection is present and the device is battery powered, the system is very patientfriendly and safe to use.

\subsection{Non-contact Electrodes}

The basic design and function of capacitive electrodes has been well explored and documented in the literature, with 

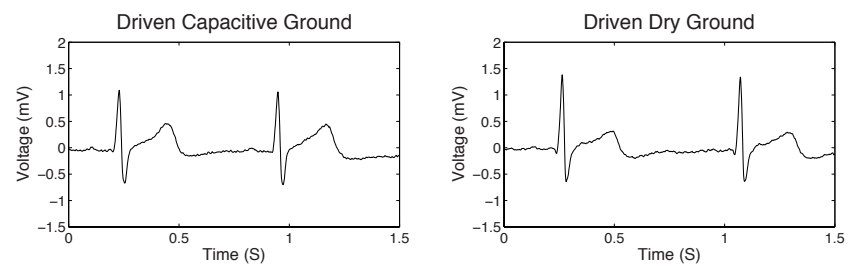

Passive Dry Ground
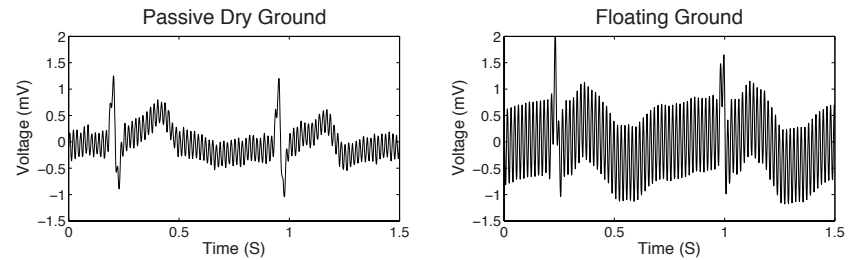

Figure 3: Comparison of different grounding techniques. The signals are shown at full bandwidth, without $60 \mathrm{~Hz}$ filters.

earliest working devices dating back to 1968 [3]. However, all commercially available devices rely on either dry or wet $\mathrm{Ag} / \mathrm{AgCl}$ electrodes, with the latter almost exclusively dominating the medical market.

Common problems associated with capacitive, non-contact electrodes include:

1. Noise - Capacitive electrodes exhibit a much larger intrinsic circuit noise floor.

2. Motion Artifacts - Even slight amounts of motion/friction saturate the signal.

3. Interference Pickup - Much greater sensitivity to $50 / 60 \mathrm{~Hz}$ line noise.

4. Complexity - The need for exotic and expensive components directly on the electrode.

In general, these problems can be categorized as arising from two sources. The first consists of circuit design, accounting for the issues with complexity and intrinsic noise. The second is largely due to mechanical implementation and account for interference and motion artifacts. Through our experience, these problems with capacitive sensors are largely solved through modern components and careful design. The details will be explained in full throughout this paper.

We have developed a simple, repeatable, robust and relatively inexpensive method for producing high quality capacitive electrodes. The physical substrate of the electrode is a standard PCB. Figure 2 shows a close up of the latest generation of our non-contact electrodes. As before, amplifier circuits to buffer the weak signal acquired electrode are housed on the top. In the center, a snap connector is used to provide mechanical stability, as well as provide compatibility with standard medical/research instrumentation (additional power, reference and ground lines are needed as well).

The bottom plate of the electrode is a solid copper fill, which forms a parallel plate capacitor with the body to couple biopotential signals. In this version, the copper fill is not insulated with soldermask, allowing the sensor to optionally function as a dry contact electrode. As a dry-contact sensor, the signal is virtually indistinguishable from clinical wet $\mathrm{Ag} / \mathrm{AgCl}$ electrodes. However, this paper will focus on the use of this sensor as a capacitive electrode, sensing signals through insulation such as fabric.

An active shield formed by the inner plane of the PCB and a ring around the sensing plate protects the electrode from external interference. The overall dimensions of the sensor is slightly larger than a US quarter.

In contrast to previous reported implementations [4] [5] which involved a combination of exotic components (such as the expensive, electrometer-grade INA116), capacitance cancellation schemes and proprietary/unpublished designs, we have built a very high quality active capacitive electrode based around a common, inexpensive and widely available amplifier, the National Semiconductor LMP7702. Only three resistors and three capacitors are required to complete the device.

The LMP7702 is a CMOS opamp in a dual SOIC-8 package with an input structure suitable for ultra-high impedance sensors. Although on paper, the current-noise is specified as ten times greater than the INA116, we have found the noise performance to be comparable, if not better in practice. In addition, the LMP7702 is specified to operate at a much lower supply voltage (down to $2.7 \mathrm{~V}$ ).

The first opamp in the package is configured as an unitygain voltage buffer. The $10 n F$ and $10 k$ resistors are used to protect the input of the amplifier and isolate the output of the amplifier from the active shield. No external input biasing network is necessary with the LMP7702 and the inputs consistently charge and stay within the rail-to-rail input range during use. Likewise, the outputs are also stable since the amplifier has rail-to-rail outputs and is configured as unity gain. This achieves optimal performance since any bias network necessarily adds noise and degrades the input impedance.

The lack of a bias network, however, results in an undefined (although full usable) DC operating point. To remove this offset as well as low frequency noise/drift, a passive RC high pass filter with a corner frequency of $0.7 \mathrm{~Hz}$ is used to center the signal around $V_{\text {ref }}$. The second opamp in the package is then buffers this high-passed signal and drives the cable connecting the electrode to the base unit. A $100 \Omega$ resistor is used to isolate the cable capacitance from the amplifier's output.

Although this lack of gain through multiple buffers is theoretically disadvantageous from a noise perspective, in practice the noise from the capacitive electrode to body interface will dominate the subsequent stages. Having a unity gain buffer also eliminates the need for precisely matched passive components at the electrode to achieve a good commonmode rejection ratio (CMRR).

As shown later, this very simple electrode design works remarkably well in practice and requires no expensive components or manufacturing processes. The use of standard PCB technology greatly simplifies the construction and allows for easy integration of the active shield [4].

\subsection{Wireless Base Unit}

Each of the electrodes outputs a buffered, unity-gain, analog signal. A compact, battery-powered base unit (Fig. 1) provides the necessary power, reference and ground lines.

An N-input differential amplifier was constructed by extending [10] the topology of the well known 3-input instrumentation amplifier. In general, any practical biopotential amplifier circuit should work well. Since the non-contact 

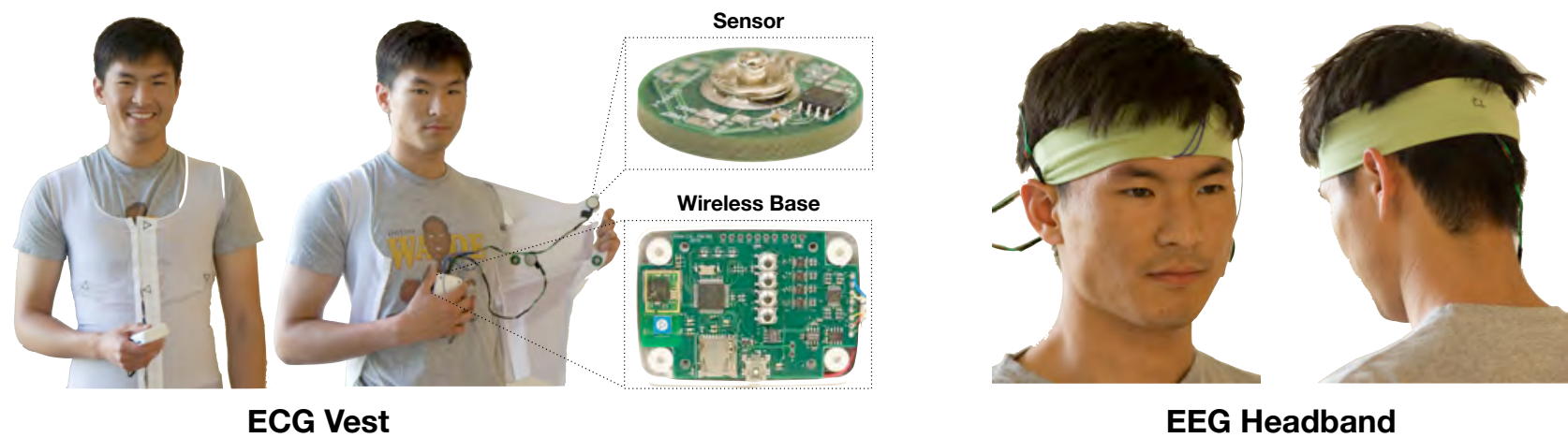

EEG Headband

Figure 4: Picture of the prototype ECG chest vest and EEG head band.

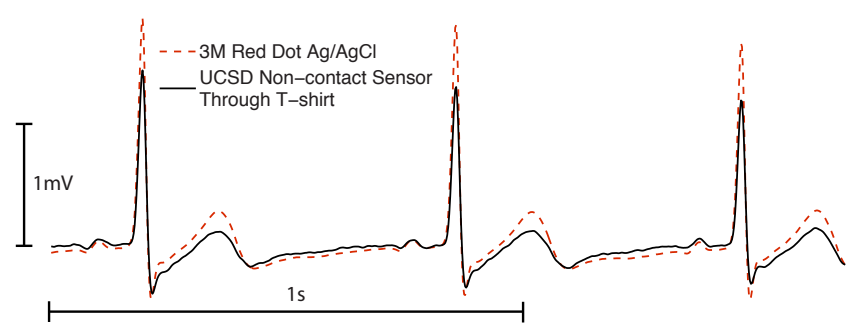

Figure 5: Detailed comparison of signal acquired simultaneously from a set of clinical grade 3M Red Dot $\mathrm{Ag} / \mathrm{AgCl}$ adhesive electrodes and the noncontact sensor. The non-contact sensor was placed over a cotton t-shirt.

electrodes are AC coupled with low-offset buffers, it was possible to incorporate a large amount of gain $(40 \mathrm{~dB}) \mathrm{di}$ rectly within one amplifier stage. A 16-bit ADC (AD7685) is used, resulting in a $\mathrm{LSB}$ of $0.5 \mu \mathrm{V}$ over an input range of $33 \mathrm{mV}$.

A two pole passive RC filter is used to filter out highfrequency components before the ADC. Both corner frequencies are set at $159 \mathrm{~Hz}$. Although the anti-alias filter provides only a shallow roll-off, we use a sufficiently high sampling rate to avoid any noticeable aliasing artifacts. This also minimizes the number of passive and active components.

The overall bandwidth of the system is then dictated by the analog high-pass filter of the capacitive electrode, $0.7 \mathrm{~Hz}$, and the antialias filter, $159 \mathrm{~Hz}$.

\subsection{Grounding}

Subject grounding is one of the most important factors in achieving good signal quality. Unfortunately, it is often overlooked and not always explained clearly, despite its vital importance. In our experience, many of the problems with non-contact sensors were due to improper grounding.

In line with the idea of a fully insulated system, we use a capacitive coupling to connect the circuit ground back to the body. A dummy electrode without components is used to as the capacitive ground electrode. Such a ground is fully insulated, but offers only a weak coupling, rendering the system susceptible to interference. Actively driven grounding schemes are a well-known technique [11] to reduce commonmode interference and have been successfully adapted for

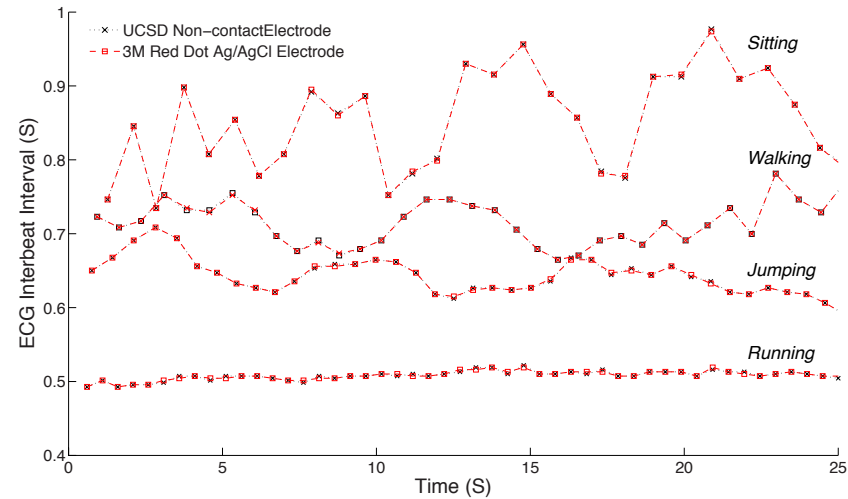

Figure 6: Extracted heart beat (R-R) intervals from the ECG signal for the $\mathrm{Ag} / \mathrm{AgCl}$ sensors versus the non-contact sensor prototype. The intervals are essentially identical.

capacitive sensors [12].

On the base unit, the common mode signal, $V_{c m}$ is connected to an inverting amplifier with gain of -100 to provide an additional $40 \mathrm{~dB}$ of CMRR for the system. The signal is fed back into the body through the dummy ground electrode.

An simple experiment, depicted in Figure. 3 illustrates the effectiveness of this active ground. The capacitive active ground is as effective as a driven dry ground contact (antistatic wrist strap). Passive dry grounds start to suffer from $60 \mathrm{~Hz}$ noise pick-up. A floating (purely parasitic) ground may also be used, but suffers from large $60 \mathrm{~Hz}$ and other low frequency artifacts, and is not suitable for any serious measurements.

The combination of the active ground and the fact that the system is battery-powered, results in a very clean signal, free from $60 \mathrm{~Hz}$ noise.

\subsection{Data Acquisition}

A simple 16-bit microcontroller (PIC24) was used to control the ADC. The base unit supports both Bluetooth wireless connectivity for live streaming of data to a $\mathrm{PC}$ or other display interface. For longer-term mobile recordings, a microSD interface is also available. The entire system is powered from a $900 \mathrm{mAh}$ rechargeable lithium-polymer battery 

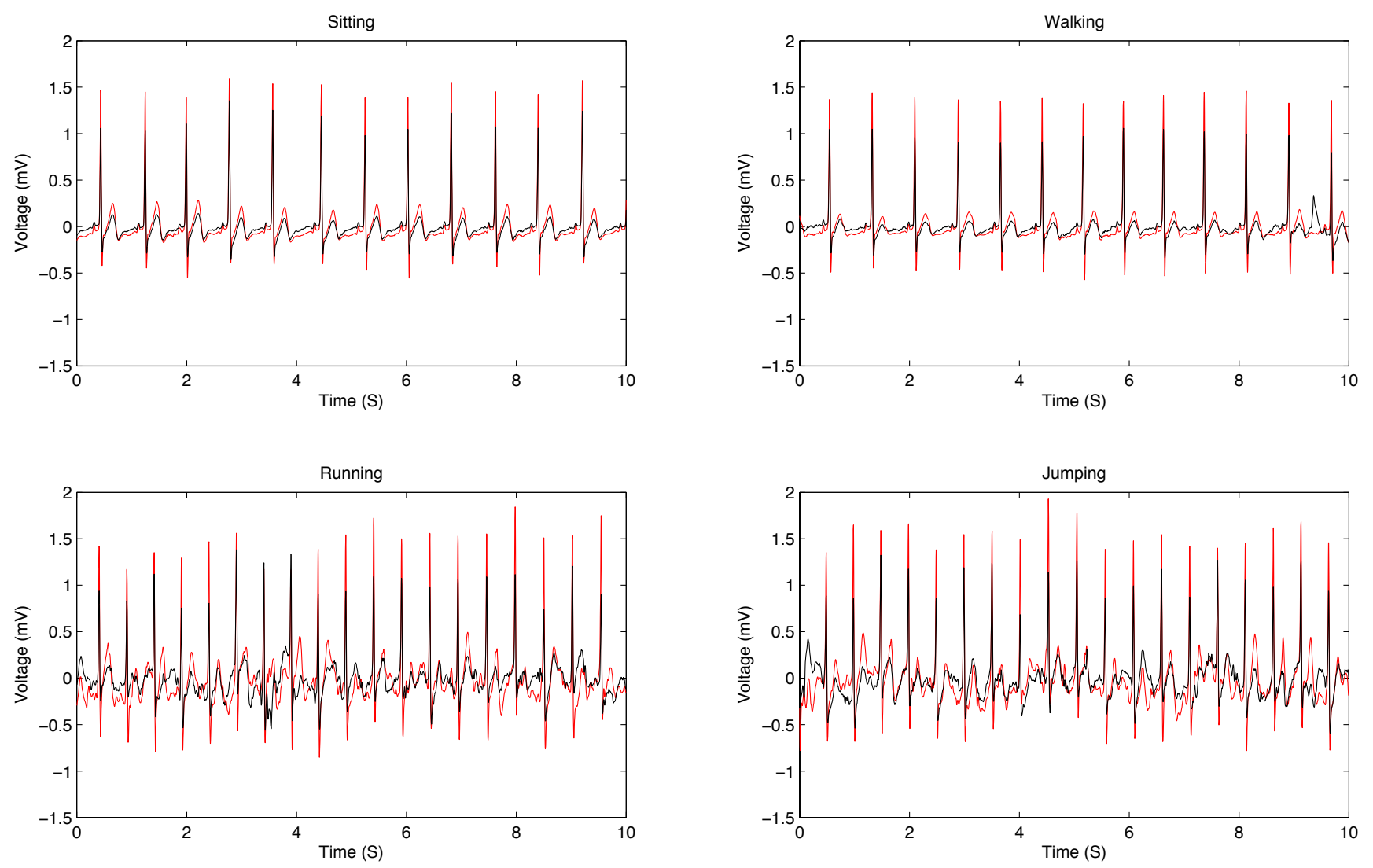

Figure 7: A 10-second long comparison of signal from 3M Red Dot (red trace) versus non-contact electrodes (black trace) during various activities. The non-contact electrodes were fixed in a tight chest band on top of a cotton shirt.

good for approximately 10 hours of continuous recording.

The device is recharged through a mini-USB connector. A USB-RS232 converter (FT232R) is also available for higher bandwidth, real-time streaming of data than what is possible with the Bluetooth application. For safety reasons, this mode should only be used with an unplugged laptop, since no power isolation circuit is provided.

For the purposes of the experiments in this paper, we utilized the Bluetooth transmitter which streamed data into a simple PC display and logging application at a rate of $343 \mathrm{~Hz}$ for the four signal channels.

For future, commercial-grade systems, it would be advantageous to use the latest in low-power wireless technology (ie. Bluetooth low-energy) as well as miniaturize the system's form-factor.

\section{WEARABLE SENSOR HARNESS}

As mentioned earlier, capacitive electrodes do not have the benefit of being fixed to body. Consequently, they are very sensitive to motion errors and require a robust enclosure to achieve an optimal signal.

We have developed an ECG chest harness and an EEG headband (Fig. 4) to mount the non-contact electrodes. As shown later, a firm enclosure that fixes the electrodes to the body allows the non-contact electrode to perform almost as well as standard adhesive $\mathrm{Ag} / \mathrm{AgCl}$ electrodes.

A compression vest was used as the basis of the ECG chest harness. Adaptation of the gynecomastia vest was prepared by sewing electrode snap connectors onto the vest and snapping the electrodes during use. Non-contact electrode placement was assigned to the two midaxillary positions. This vest was ideal for electrode placement due to its elastic contouring ability, covering of the thoracic surface with sufficient firmness. A second elastic band was also optionally available to add additional security for holding the electrodes in place.

For EEG experiments, a simple, tight, elastic cloth headband was used in a similar fashion by sewing in snap connectors for the non-contact electrodes. In contrast to known commercial and research headbands, our version allows for signals to be acquired through hair using the capacitive electrodes. The design and operation of the EEG headband was especially challenging due to the flexible properties of hair which make securing the electrodes difficult.

\section{PHYSIOLOGICAL DATA}

The ECG vest and EEG headband were used to collect live data using the capacitive sensors. For the purposes of generating a direct comparison, two of the four electrode inputs were connected to standard passive $\mathrm{Ag} / \mathrm{AgCl}(3 \mathrm{M}$ Red Dot) and the other two were connected to the capacitive active electrodes. The subject was a healthy 21 year old male. Experiments were conducted in a standard electrical engineering lab with no effort to eliminate sources of 

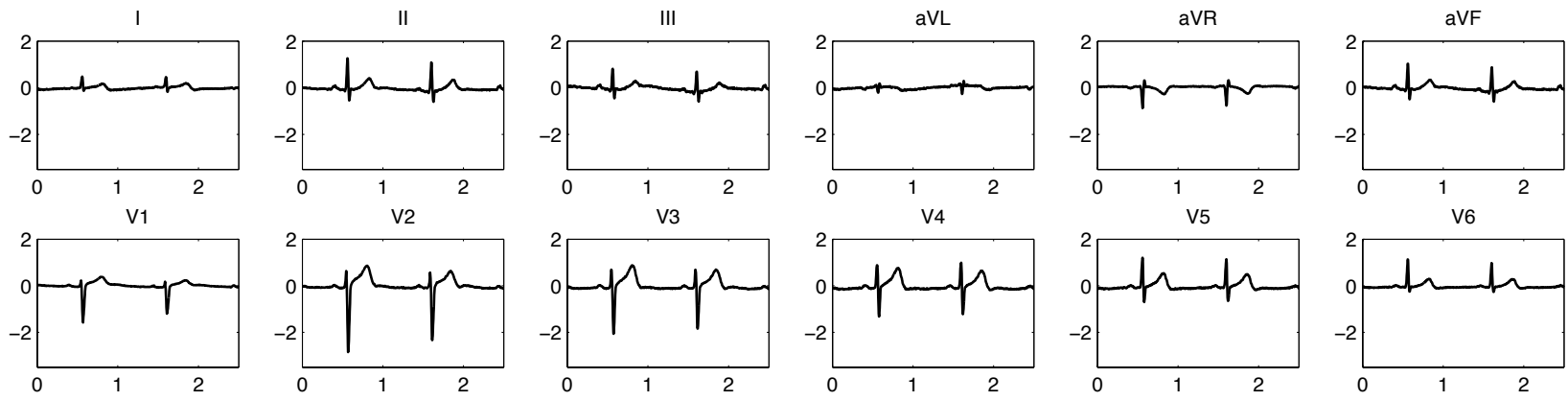

Figure 8: Derived 12-lead ECG from 4-electrode chest band in an EASI array.

interference.

\subsection{ECG Experiments}

For the ECG tests, the capacitive electrodes were mounted into the tight, body-fitting harness with the two capacitive electrodes on the left and right sides of the ribcage. The subject wore a simple cotton t-shirt underneath the harness. Two $\mathrm{Ag} / \mathrm{AgCl}$ adhesive electrodes were also placed in a nearby position directly on the skin. The output signal for the capacitive sensor was defined by taking the difference between the two and likewise of the $\mathrm{Ag} / \mathrm{AgCl}$ electrode pair.

Figure 5 shows a detailed plot of a ECG sample taken while the subject was sitting at rest. The overall signals are nearly indistinguishable in both shape and noise levels, even though the capacitive electrodes were operating through clothing. All relevant ECG features are clearly visible. The slightly smaller amplitude of the signal from the non-contact electrode is likely due to the signal attenuation from the extremely high source impedance.

One previous study [6] compared the performance of a proprietary capacitive electrode design versus contact electrodes with a subject lying down. We extend the methodology established in these tests to include data with an actively moving subject. Figure 7 shows 10-second plots comparing the signal acquired from the capacitive and $\mathrm{Ag} / \mathrm{AgCl}$ electrodes.

As expected, the signal remains mostly undisturbed while the subject is at rest and walking lightly. During more vigorous activities, motion artifacts become problematic for both electrodes types, rendering the ECG signal useful for only $\mathrm{R}-\mathrm{R}$ beat detection. The signal for the capacitive electrode is not substantially worse, as long as the capacitive electrodes are fixed tightly against the body using the harness. It should be worth noting, however, that capacitive electrodes are extremely sensitive to friction (rubbing against cotton), which necessitates the hight chest harness.

We again used the same methodology as [6] to compare the extracted R-R beat intervals to show the equivalence of $\mathrm{Ag} / \mathrm{AgCl}$ and capacitive electrodes, extending their study to subjects in motion. The plot is shown in Figure 6 . The extracted $\mathrm{R}-\mathrm{R}$ beat intervals are virtually identical across all the tested activities.

For applications beyond simple heart beat and rhythm detection, a clinical-grade multi-lead signal is required. We used the four input channels available from our device to construct an EASI [13] array to obtain a derived 12-lead
ECG signal. The positioning and transformation coefficients were taken from [13].

Placement of electrodes at standard EASI positions and ground was accomplished by use of the adapted gynecomastia vest. Figure 8 shows the derived 12-lead ECG, which show the expected shape and features, such as the increasing amplitude of the R-wave progressing from the V1 to V6 lead.

\subsection{EEG Experiments}

Similar to the cardiac data, two of the input channels were connected to $\mathrm{Ag} / \mathrm{AgCl}$ adhesive electrodes to achieve a direct comparison with the non-contact sensors. For the EEG experiments, one capacitive electrode was placed on the forehead (Fp1) and the second on the back (Oz), through hair. Likewise, an $\mathrm{Ag} / \mathrm{AgCl}$ electrode was also placed on the forehead (Fp2). However, since it was not possible to place an contact electrode through hair, the second $\mathrm{Ag} / \mathrm{AgCl}$ electrode was placed on the mandible (A1). Unless specified, the output for each electrode was defined as the signal at each channel minus the common-mode of four channels.

Figure 9 shows a detailed time-domain plot of all four EEG channels with the subject relaxing, at rest with closed eyes. Not only are the expected alpha rhythms clearly visible (especially in the occipital electrode), the signals from the $\mathrm{Ag} / \mathrm{AgCl}$ and capacitive electrodes are virtually identical. Strong alpha wave activity is seen, as expected for an awake, relaxed subject. The signals are all shown at full bandwidth, without an additional digital filtering showing the effectiveness of the active shield and driven ground.

A second montage consisting of two signals was obtained by using the $\mathrm{A} 1 \mathrm{Ag} / \mathrm{AgCl}$ electrode as a reference for the frontal and parietal capacitive electrodes. In this experiment, the subject was asked to blink several times and then close his eyes. Figure 10 shows the clear blink artifacts in the Fp1A1 signal followed by the onset of alpha waves in the OzA1 signal.

The ability to easily obtain signals from the normally hair covered occipital and parietal regions is especially useful for brain-computer (BCI) and other EEG-based neural interfaces. Systems which can only obtain frontal EEG activity cannot obtain important responses including the P300 evoked potential and the stead-state visual evoked potentials (SSVP), commonly used in BCI applications.

To test the effectiveness of the capacitive electrode over the haired occipital region, a simple experiment where the subject was asked to watch a flashing LED was devised to 


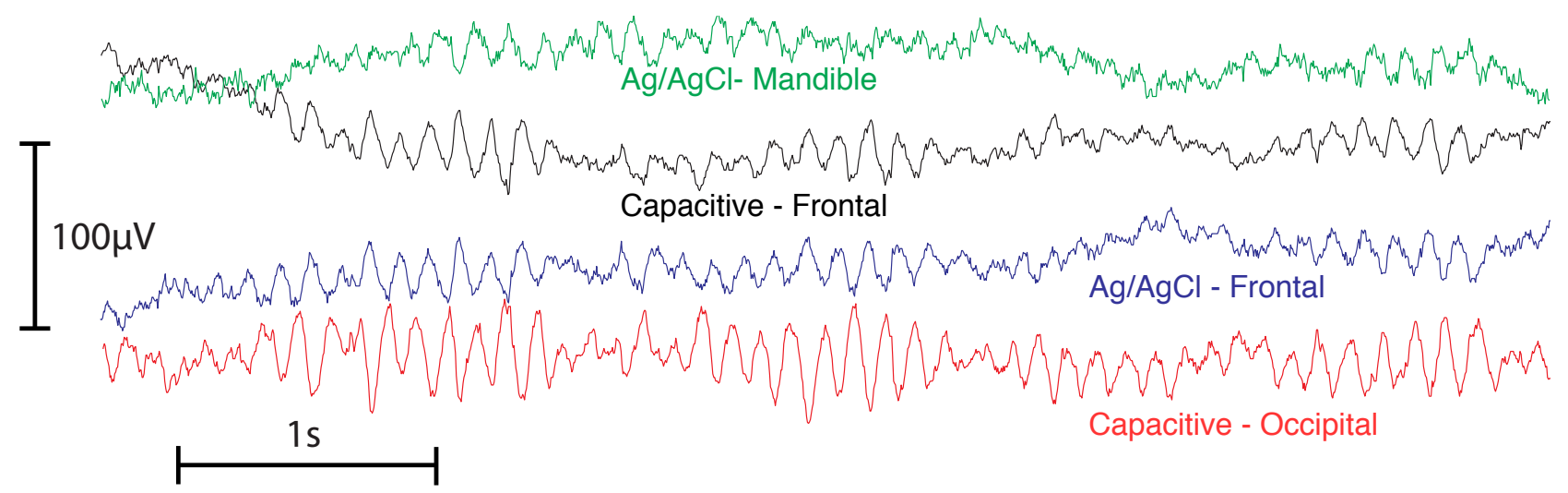

Figure 9: Close-up of EEG signals acquired using a mix of Ag/AgCl (3M Red Dot) and capacitive non-contact electrodes. No extra filtering beyond the analog anti-alias has been applied to the raw signal to show that the sensor is free from $60 \mathrm{~Hz}$ interference.

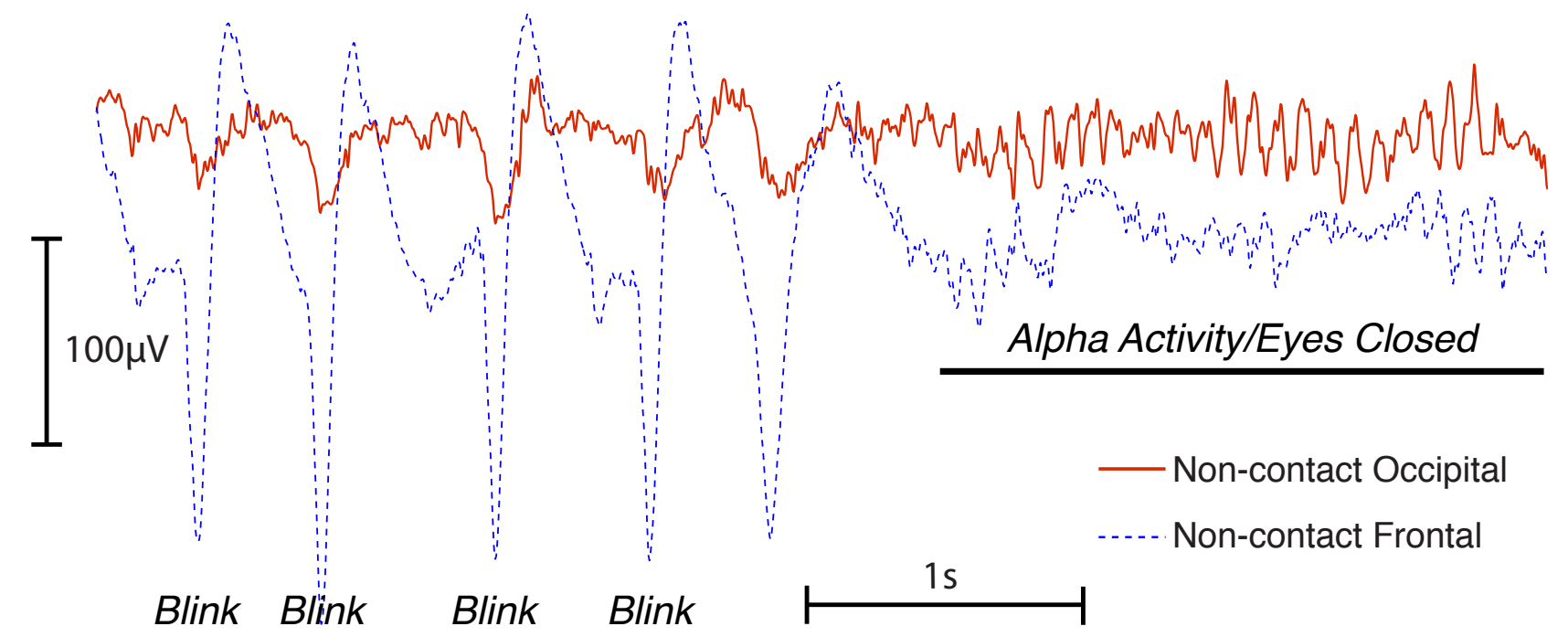

Figure 10: Experiment showing the signal from the frontal capacitive electrode (Fp1A1) in blue and the signal from the occipital capacitive electrode (O1A1). Eye blink artifacts are visible in the frontal electrodes during the first half of the recording. Strong alpha activity is seen in the occipital electrode after the subject's eyes close.

measure the SSVP response.

Sleep diagnosis is an important medical application of EEG technology, and one that will directly benefit from having easy to use, unobtrusive, non-contact sensors. Home sleep monitoring and coaching systems are already commercially available but rely on the limited set of signals available from forehead contact electrodes. The non-contact sensor allows for EEG signals to be acquired from the entire head, without gels.

A spectrogram depicting a period of sleep EEG taken from the O1 capacitive electrode is shown in Figure. 11. The subject was asked to take a short nap. The different frequency components of sleep EEG activity are visible in the timefrequency plot.

\section{CONCLUSION}

We present the full designs for a wireless ECG/EEG monitoring system using insulated, non-contact sensors.
Non-contact sensors have traditionally been thought of as unsuitable for medical-grade applications, but we demonstrate how careful design, both at the circuit and system level can produce signals comparable to clinical grade $\mathrm{Ag} / \mathrm{AgCl}$ electrodes for both ECG and EEG applications. The one area that is still currently unaddressed, however, is sensitivity to motion artifacts. Efforts directed at mitigating the non-contact electrode's inherent sensitivity to motion/friction effects at the system and signal processing level is expected to yield large returns for this field.

This wireless and wearable system is ideally suited for future mobile health applications by being much more comfortable and patient-friendly than traditional contact based systems.

\section{ACKNOWLEDGEMENTS}

This work was generously supported by National Semiconductor and NSF SBE-0847752. The authors thank Dong-mei 


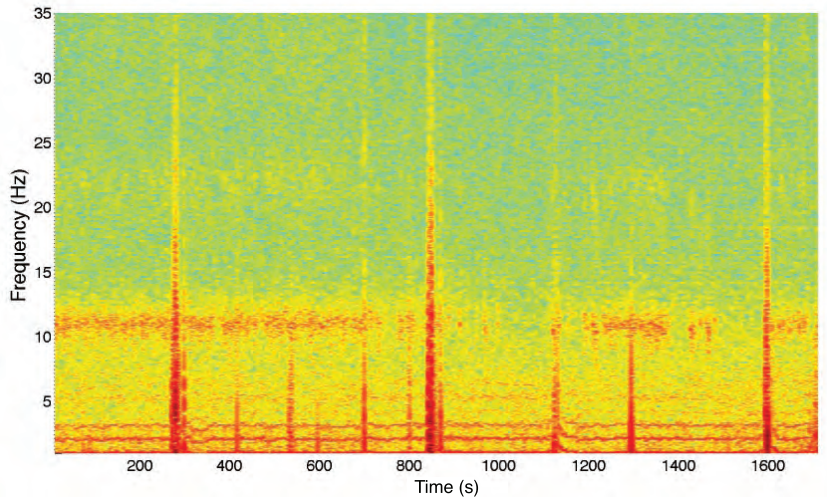

Figure 11: Spectrogram of sleep EEG taken with the headband. The signal is taken from the non-contact electrodes (Fp1O1).

Yan and Mehmet Alsan at National for their support and fruitful discussions. We also thank Siddharth Joshi for help with setting up experimentation.

\section{REFERENCES}

[1] T.J. Sullivan, S.R. Deiss, Tzyy-Ping Jung, and G. Cauwenberghs. A brain-machine interface using dry-contact, low-noise eeg sensors. In Circuits and Systems, 2008. ISCAS 2008. IEEE International Symposium on, pages 1986 -1989, 18-21 2008.

[2] Emil Valchinov and Nicolas Pallikarakis. An active electrode for biopotential recording from small localized bio-sources. BioMedical Engineering OnLine, $3(1): 25,2004$.

[3] Alfredo Lopez and Philip C. Richardson. Capacitive electrocardiographic and bioelectric electrodes. Biomedical Engineering, IEEE Transactions on, BME-16(1):99 -99, jan. 1969.

[4] T.J. Sullivan, S.R. Deiss, and G. Cauwenberghs. A low-noise, non-contact eeg/ecg sensor. In Biomedical Circuits and Systems Conference, 200\%. BIOCAS 200\%. IEEE, pages 154-157, 27-30 2007.

[5] C J Harland, T D Clark, and R J Prance. Electric potential probes - new directions in the remote sensing of the human body. Measurement Science and Technology, 13(2):163, 2002.

[6] J.M. Lee, F. Pearce, C. Morrissette, A.D. Hibbs, and R. Matthews. Evaluating a capacitively coupled, noncontact electrode for ecg monitoring. Sensors Magazine, 2005.

[7] Chulsung Park, P.H. Chou, Ying Bai, R. Matthews, and A. Hibbs. An ultra-wearable, wireless, low power ecg monitoring system. In Biomedical Circuits and Systems Conference, 2006. BioCAS 2006. IEEE, pages $241-244$, nov. 2006.

[8] Yu.M. Chi, S.R. Deiss, and G. Cauwenberghs. Non-contact low power eeg/ecg electrode for high density wearable biopotential sensor networks. In Wearable and Implantable Body Sensor Networks, 2009. BSN 2009. Sixth International Workshop on, pages 246-250, 3-5 2009.

[9] Yu.M. Chi and G. Cauwenberghs. Micropower non-contact eeg electrode with active common-mode

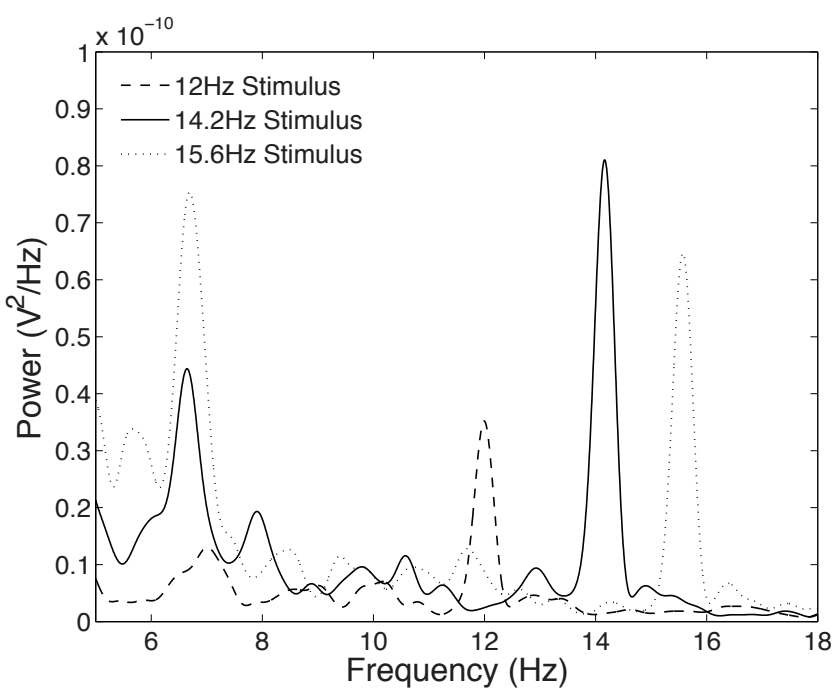

Figure 12: Power power spectrum from the SSVP experiment. The subject was asked to look at a flashing stimulus at specific frequencies. The same frequency can be observed in the occipital capacitive electrode.

noise suppression and input capacitance cancellation. In Engineering in Medicine and Biology Society, 2009. $E M B C$ 2009. Annual International Conference of the IEEE, pages 4218 -4221, 3-6 2009.

[10] A. Peper A.C. MettingVanRijn and C.A. Grimbergen. Amplifiers for bioelectric events: A design with a minimal number of parts. Medical and Biological Engineering and Computing, 1994.

[11] Bruce B. Winter and John G. Webster. Driven-right-leg circuit design. Biomedical Engineering, IEEE Transactions on, BME-30(1):62 -66 , jan. 1983.

[12] Ko Keun Kim, Yong Kyu Lim, and Kwang Suk Park. Common mode noise cancellation for electrically non-contact ecg measurement system on a chair. In Engineering in Medicine and Biology Society, 2005. IEEE-EMBS 2005. 27th Annual International Conference of the, pages 5881-5883, 17-18 2005.

[13] Dirk Q. Feild, Charles L. Feldman, and B. Milan Hor[aacute][ccaron]ek. Improved easi coefficients: Their derivation, values, and performance. Journal of Electrocardiology, 35(4, Part 2):23 - 33, 2002.

[14] A.J. Clippingdale, R. J. Clark, and C Watkins. Ultrahigh impedance capacitively coupled heart imaging array. Review of Scientific Instruments, 65(1):269-270, 1994.

[15] R. Matthews, N.J. McDonald, P. Hervieux, P.J. Turner, and M.A. Steindorf. A wearable physiological sensor suite for unobtrusive monitoring of physiological and cognitive state. In Engineering in Medicine and Biology Society, 2007. EMBS 2007. 29th Annual International Conference of the IEEE, pages 5276 -5281, 22-26 2007.

[16] A. Ueno, Y. Akabane, T. Kato, H. Hoshino, S. Kataoka, and Y. Ishiyama. Capacitive sensing of electrocardiographic potential through cloth from the 
dorsal surface of the body in a supine position: A preliminary study. Biomedical Engineering, IEEE Transactions on, 54(4):759 -766, april 2007.

[17] M Oehler, V Ling, K Melhorn, and M Schilling. A multichannel portable ecg system with capacitive sensors. Physiological Measurement, 29(7):783, 2008.

[18] T. Maruyama, M. Makikawa, N. Shiozawa, and Y. Fujiwara. Ecg measurement using capacitive coupling electrodes for man-machine emotional communication. In Complex Medical Engineering, 200\%. CME 200\%. IEEE/ICME International Conference on, pages 378 -383, 23-27 2007.

[19] Tadayuki Matsuo, Kazuhiro Iinuma, and Masayoshi Esashi. A barium-titanate-ceramics capacitive-type eeg electrode. Biomedical Engineering, IEEE Transactions on, BME-20(4):299 -300, july 1973.

[20] A. Aleksandrowicz and S. Leonhardt. Wireless and non-contact ECG measurement system- the Aachen SmartChair. ActaPolytechnica, 2:68-71, June 2007.

[21] Jin-Chern Chiou, Li-Wei Ko, Chin-Teng Lin, Chao-Ting Hong, Tzyy-Ping Jung, Sheng-Fu Liang, and Jong-Liang Jeng. Using novel mems eeg sensors in detecting drowsiness application. In Biomedical Circuits and Systems Conference, 2006. BioCAS 2006. IEEE, pages $33-36$, nov. 2006.

[22] A Searle and L Kirkup. A direct comparison of wet, dry and insulating bioelectric recording electrodes. Physiological Measurement, 21(2):271, 2000.

[23] C J Harland, T D Clark, N S Peters, M J Everitt, and P B Stiffell. A compact electric potential sensor array for the acquisition and reconstruction of the 7-lead electrocardiogram without electrical charge contact with the skin. Physiological Measurement, 26(6):939, 2005. 